\title{
A Soft Magnetic Core Can Enhance Navigation Performance of Magnetic Nanoparticles in Targeted Drug Delivery
}

\begin{tabular}{|r|l|}
\hline Journal: & Transactions on Mechatronics \\
\hline Manuscript ID & TMECH-04-2017-6570 \\
\hline Date Submitted by the Author: & 11-May-2017 \\
\hline Complete List of Authors: & $\begin{array}{l}\text { Zhang, Xingming; Gyeongsang National University, School of Mechanical } \\
\text { and Aerospace Engineering; Harbin Institute of Technology at Weihai } \\
\text { Le, Tuan-Anh ; Gyeongsang National University, School of Mechanical and } \\
\text { Aerospace Engineering } \\
\text { Hoshiar, Ali; Gyeongsang National University, School of Mechanical and } \\
\text { Aerospace Engineering; Islamic Azad University } \\
\text { Yoon, Jungwon; Gyeongsang National University, School of Mechanical and } \\
\text { Aerospace Engineering }\end{array}$ \\
\hline Keywords: & $\begin{array}{l}\text { Magnetic resonance imaging, Medical Image Registration and Navigation, } \\
\text { Drug delivery systems, Nanotechnology }\end{array}$ \\
\hline Are any of authors IEEE & Yes \\
\hline Member?: & Are any of authors ASME \\
Member?: & No \\
\hline \multicolumn{2}{|c|}{} \\
\hline $\begin{array}{l}\text { Note: The following files were submitted by the author for peer review, but cannot be converted to } \\
\text { PDF. You must view these files (e.g. movies) online. }\end{array}$ \\
\hline 1D-Navigation of MNPs.mp4
\end{tabular}




\title{
A Soft Magnetic Core Can Enhance Navigation Performance of Magnetic Nanoparticles in Targeted Drug Delivery
}

\author{
Xingming Zhang ${ }^{1,2, a)}$, Tuan-Anh Le ${ }^{2, a)}$, Ali Kafash Hoshiar ${ }^{2,3}$ and Jungwon Yoon ${ }^{2, b)}$ \\ ${ }^{1}$ School of Naval Architecture and Ocean Engineering, Harbin Institute of Technology at Weihai, Weihai, Shandong, China. \\ ${ }^{2}$ School of Mechanical and Aerospace Engineering \&ReCAPT, Gyeongsang National University, Jinju 660-701, Republic of Korea, \\ ${ }^{3}$ Faculty of Industrial and Mechanical Engineering, Islamic Azad University, Qazvin Branch, Qazvin, Iran, 34199-15195 \\ a) Xingming Zhang and Tuan-Anh Le contributed equally to this work as first author. \\ b) Corresponding author: jwyoon@gnu.ac.kr
}

\begin{abstract}
Magnetic nanoparticles (MNPs) are a promising candidate for carriers in drug delivery systems, and a navigation system with real-time actuation and monitoring of MNPs is inevitably required for more precise targeting and diagnosis. The major challenge for MNP navigation is achieving a high magnetic field gradient, which can provide a higher steering force with enhanced monitoring resolution. A soft magnetic core with coils can increase the magnetic gradient field; however, this also generates harmonic noise, which makes obtaining MNP monitoring signals with magnetic particle imaging (MPI) difficult. To allow both MPI and electromagnetic actuator functions with magnetic cores in real time, a novel navigation system with a coil-core structure for an MPI scheme is suggested, which is less sensitive to the core and has a higher magnetic steering force. Based on the new MPI scheme and soft magnetic cores, the proposed navigation system can implement one-dimensional MNP navigation and two-dimensional MPI with a higher steering force and enhanced image resolution.
\end{abstract}

Index Terms-Soft Magnetic Core, Magnetic Particle Imaging, Navigation Resolution, Magnetic Nanoparticles

\section{INTRODUCTION}

$M$ agnetic nanoparticles (MNPs) that can be manipulated using magnetic fields can function at both the cellular and the molecular levels [1], making them suitable for use as carriers for targeted drug delivery and contrast agents in magnetic particle imaging (MPI) [2]. Although drugs reach their target locations, they also affect healthy tissues, causing undesirable side effects. Thus, targeted drug delivery (TDD) systems have been developed to enhance treatment efficiency and maintain optimal doses at a desired location [3]. Our recent work demonstrated the effectiveness for the delivery of MNPs using an electromagnetic actuator into the brains of mice based on an open-loop control approach using extensive in vivo experiments [4]. However, tracking of the nanoparticles in TDD will be inevitable for more precise targeting and diagnosis with spatial information of nanoparticles.

Several methods have been suggested for developing a TDD with a feedback scheme, such as using ultrasound to locate solid micro-size particles [5] or using a microscope to track visible particles [6]. The groundbreaking concept of the magnetic resonance navigation (MRN) system includes a feedback control scheme proposed by Martel et al. [7, 8]. In MRN, embedded micro-particles are tracked by magnetic resonance imaging (MRI) systems and controlled by additional electromagnetic coils.

MPI is a fast and sensitive imaging modality for measuring the spatial distribution of MNPs [2]. MPI systems offer spatial resolutions on the millimeter scale and high temporal resolutions, with about 45 three-dimensional volumes/s [2]. In addition, the detection threshold of MNPs is less impaired by background signals from the host tissue compared to MRI. Tracer magnetic nanoparticles in MPI can provide spatial information and be used as drug carrier particles. When adopting MNPs of 50-nm core size with a $5.1 \mathrm{~T} / \mathrm{m} / \mu_{0}$ magnetic field gradient, potential resolutions of up to $0.11 \mathrm{~mm}$ can be achieved [9]. Therefore, a real-time navigation system for MNPs based on MPI is capable of performing actuation and monitoring tasks simultaneously. This has particular application in precise steering and tracking of MNPs with real-time feedback schemes for drug delivery to the brain.

The control performance of MPI-based navigation systems mainly depends on the magnetic field gradient of both the electromagnetic actuator (EMA) and the MPI. The former determines the magnitude of the steering force for manipulation of nano-sized particles and the latter the resolution of the MPI image. Cherry et al. [10] showed that the magnetic force acting on MNPs using standard magnetic actuation systems cannot deliver MNPs to brain cells due to the nanosize of these particles. For specified nanoparticles for monitoring, the full-width at half-maximum (FWHM) resolution of MPI is proportional to the reciprocal of the gradient [9]; a larger magnetic field gradient should be considered to increase both the steering force and the image quality for MNP monitoring. However, existing MPI schemes with only coil topology have some limitations:

1. One major obstacle limiting MPI upscaling is the dramatic reduction in the magnetic field strength and the magnetic gradient [11], which is disproportionate to the cube of the distance.

2. In the original MPI concept, the frequency of the driving field varied in the range from $10 \mathrm{kHz}$ up to about $100 \mathrm{kHz}$, with a peak amplitude from around $10 \mathrm{mT} / \mu_{0}$ up to about $100 \mathrm{mT} / \mu_{0}$ [11]. These high frequencies and amplitudes impose a severe restriction on upscaling the system for the human body. Meanwhile, as the human body is conductive, the applied time-varying magnetic fields induce eddy currents, which can increase the probability of unpleasant peripheral nerve 
stimulation (PNS) [12]. Consequently, the amplitude and frequency for MPI should be within safe ranges for humans. Keeping the magnitude and frequency within safe limits and providing a large field of view (FOV) are in opposition and present a design limitation for MPI.

3. MNPs exposed to a high-frequency and high-amplitude field generate higher harmonic signals of an excitation frequency, which is useful information in the original MPI scheme. However, the wider harmonic signal requires a wide-bandwidth receiver coil [13], which also necessitates the installation of a highly precise and fast response circuit with expensive and bulky components.

One typical method for increasing the gradient field is to increase the size of the coil or to use superconductivity. However, a compact and efficient way to increase the gradient field is utilization of a soft magnetic material core. Soft magnetic cores, which are easily magnetized and demagnetized, can enhance the field strength and gradient [14]. Latulippe et al. [15] proposed the concept of dipole field navigation, which utilizes ferromagnetic cores in an MRI scanner to generate high field gradients. However, soft magnetic materials exhibit a nonlinear magnetization response and are not suitable for the original MPI scheme. Moreover, a soft magnetic material exposed to a high-frequency and high-amplitude magnetic field generates a harmonic distortion, which will screen the particles' signal during the MPI process. Even if the soft cores are isolated with shielding plates, a large reduction in the drive field for the generation of particle signals will occur. In this paper, we introduce a novel navigation system for MNPs based on an MPI system enhanced by soft magnetic cores. The proposed system can provide a feedback scheme for MNPs. In the proposed MPI system, we utilize a low-frequency field (called the low-frequency drive field in this paper) to move the field-free point (FFP), and employ a low-amplitude field at high frequency (called the excitation field in this paper) to oscillate the magnetization near the FFP. As the amplitude of the received signal is used to reconstruct the MPI images, the proposed MPI system, called Amplitude Modulation MPI (AM MPI), has the following three major advantages through the use of soft magnetic cores:

1. The low-amplitude excitation field combined with the low-frequency drive field utilizes the iron cores available to the MPI function with a higher gradient field.

2. Since the low-amplitude excitation field has a $40 \mathrm{kHz}$ frequency with an amplitude of $200 \mu \mathrm{T}$, the possibility of PNS is significantly reduced.

3. AM MPI only requires a narrow-band receiver coil for easy impedance match design and elimination of noise from other frequencies.

Therefore, the proposed method can enhance both image quality and the steering magnetic force for higher manipulability of a navigation system for MNPs, using soft magnetic cores. In this paper, we propose an MPI-based one-dimensional (1D) navigation system with iron cores. The effects of iron cores on EMA and MPI periods are discussed in detail and the proposed MPI-based navigation system is demonstrated through 1D guidance tests to show that the iron cores can enhance the steering force of MNPs. Finally, a mechanical scanner is used to demonstrate that a 2D map of MNP distribution exhibits better image quality.

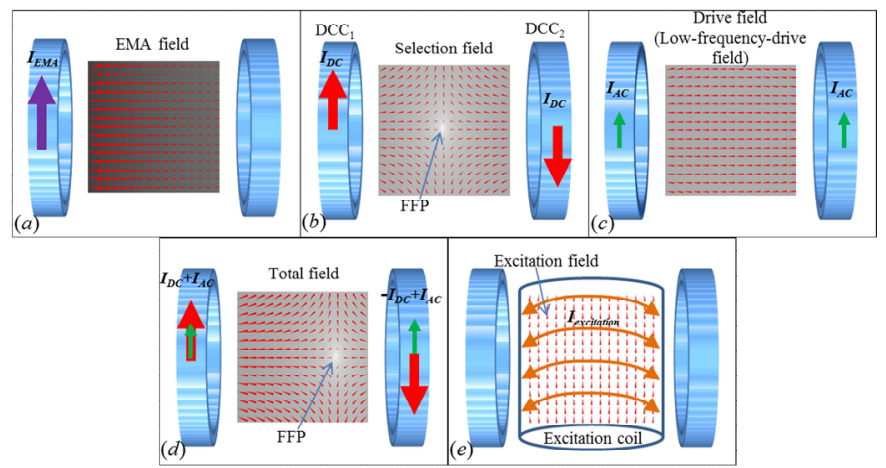

Fig. 1 The functional magnetic fields generated by different currents in workspace. The grayscale and the length of the cones represent the magnitude field strength, while the direction of cone indicates the field direction. (a) During the EMA period, the EMA field, which is a gradient field used to steer the MNPs, is induced by current $I_{E M A}$ in the DCC. (b) An FFP is located at the center of the selection field, which is generated by current $I_{D C}$ in the opposite direction. (c) The drive field is a homogeneous field generated by same-direction currents $I_{A C}$ in the coils. (d) The effect of $I_{D C}$ and $I_{A C}$ results in the movement of the FFP. (e) The excitation field is generated by the excitation coil with a high-frequency current $I_{\text {excitation }}$; because of the small amplitude compared to the selection and drive fields, the position of the FFP can be seen as unrelated to the excitation field.

\section{NAVIGATION SYSTEM OF MNPS}

In our previous work [16][17], we introduced a hybrid navigation system concept comprised of an EMA mode and an MPI mode. The coil topology was composed of air-core coils as shown in Fig. 1(a). In EMA mode, one of the differential-current coil (DCC) [14] loads the current $I_{E M A}$ to generate the magnetic field gradient (Fig. 1a), which can steer the MNPs towards the current-carrying coil. In MPI mode, the DCCs load two currents $I_{D C}$ that have equal amplitude but opposite directions to generate the selection field as well as an FFP (Fig. 1b). The FFP shift is achieved by the superposition of the static selection field and a time-varying drive field; the drive field induced by $I_{A C}$ is spatially homogeneous at any one moment (Fig. 1c and d). The DCCs combine each coil for the low-frequency drive field and for the selection field in one set of coils. The excitation field is employed to oscillate the magnetization of the particles and then to generate the signal, as shown in Fig. 1(e).

Real-time MPI and EMA in the proposed navigation system can be achieved by applying a time-division multiplexing scheme to the coil topology [16], which can integrate the EMA and MPI tasks through time sequencing. Every navigation period consists of at least one MPI scanning period to detect MNP positions and one EMA period to control MNP positions. During the EMA mode, magnetic fields will be high enough to steer MNPs, which results in MNP saturation. However, the MNPs must be demagnetized before MPI mode. Furthermore, owing to the high impedance of coils, the currents in coils cannot be changed immediately between EMA and MPI modes. Therefore, a relaxation period is introduced for demagnetization of MNPs and prevention of a voltage surge from the coils. During MPI mode, the alternating magnetic field 


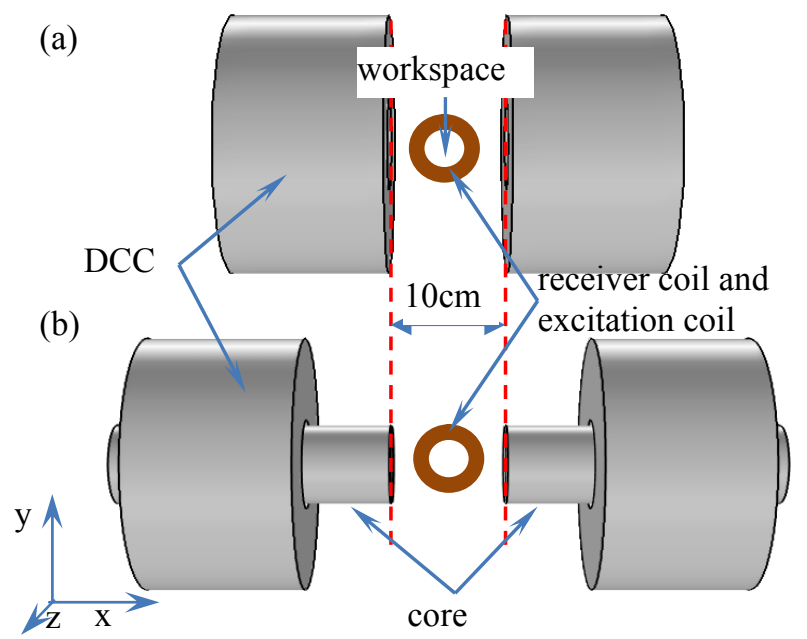

Fig. 2 (a) An air-core coil navigation system, the DCCs are the hollow coils. (b) A core-coil navigation system; an iron core is installed in the hollow coils. Both systems have the same combination of a signal receiver coil and a field excitation coil, which are placed between the DCCs. The size of the workspaces and the gap between DCCs are set to the same value: $4 \mathrm{~cm}$ and $10 \mathrm{~cm}$, respectively. Each cylindrical core is $19.5 \mathrm{~cm}$ in length and $6 \mathrm{~cm}$ in diameter.

may affect MNP positions; however, our previous work demonstrated that the force factor during the MPI cycle has little effect on the position of MNPs compared to motion during EMA mode [17] due to the alternating characteristics. Therefore, MPI mode operates during an off-cycle period in the actuation duty cycle for navigation control.

Since each gradient field in EMA and MPI mode plays a key role in enhancing navigation performance, in this paper, we propose the installation of a soft core into the hollow coil in an existing navigation system, as shown in Fig. 2. The gradient fields of a navigation system with iron cores are compared with a navigation system with air cores in the subsequent chapters. In Fig. 2, the center of the signal receiver coil is defined as the coordinate origin, the axis of the DCCs is the $x$-axis, and the axis of the receiver coil is the $z$-axis.

\section{IRON CORE FOR MAGNETOPHORETIC FORCE}

The MNPs in the workspace are saturated and steered by a gradient magnetic field, which is induced by $I_{E M A}$ during the EMA period, as shown in Fig. 1(a). The magnetophoretic force on MNPs is given by:

$$
F_{m}=\mu_{0} v_{p} M_{s} \cdot \nabla H
$$

where $\mu_{0}$ is the vacuum permeability, $v_{p}$ is the core volume of MNPs, $M_{s}$ is the situation magnetization, and $\nabla H$ is the gradient of the magnetic field. The saturation force is proportional to the gradient, which can be enhanced by the iron core. For magnetite, the most commonly used material in MNPs, a magnetic intensity of about $1.63 \mathrm{~T}$ is needed to saturate the magnetization. However, owing to the non-linear shape of the magnetization, a lower magnetic field of $0.126 \mathrm{~T}$, which is only $8 \%$ of the magnetic intensity required for saturation, can magnetize the magnetite until $74 \%$ of the saturated magnetization [14]. Therefore, achieving this medium range for the magnetic field can generate sufficient magnetization in the particles; then, generation of a higher gradient will be more effective. Because an increased number of turns and/or current in the coils to induce the higher gradient field requires larger and heavier coils, using the cores inside the coils to amplify the magnetic field is a compact and energy-efficient method.

To improve the efficiency of the electromagnets for MNP guidance, structural parameters of the cores and coils were chosen based on simulation results to provide the highest magnetic force in the region of interest, which was set at the size of a mouse brain [14]. We found that increasing the width or height of the coil increased the magnetic field strength and gradient. It was also found that the wire diameter affected the optimum design. Investigations of core parameters demonstrated that the distance between cores influenced both the magnetic field strength and gradient, whereas the minimum radius of the cores affected only the gradient and its impact on the magnetic strength was minor. Moreover, the effect of tip length on both the magnetic field intensity and gradient was negligible. Certain constraints should also be considered in the system design. The output power supply and inner and outer coil diameters should be considered to be constraints. Based on these constraints and the simulation results, the optimum configuration was chosen to satisfy the largest minimum force factor in the region of interest (ROI) [14] (see Figure 2b). Then, a cylindrical core structure can significantly strengthen the magnetic field and its gradient at the ROI by about tenfold of the force factor.

\section{IRON-CORE-COMPATIBLE MPI SCHEME}

\section{A. Signal produced by the original MPI}

The original MPI scheme relies on the nonlinearity of the MNP magnetization curves, with the MNP magnetization saturated with the saturation field strength. The relationship between the magnetization of the particles and the outer magnetic field can be described using Langevin theory of paramagnetism [2]:

$$
M=c v_{p} M_{s} L\left[\frac{m H}{k_{B} T}\right]=c v_{p} M_{s}\left[\operatorname{coth}\left(\frac{m H}{k_{B} T}\right)-\frac{k_{B} T}{m H}\right]
$$

where $L$ is the Langevin function, in particular $L(0)=0, M_{S}$ is the saturation magnetization of the particle, $v_{p}$ is the volume of core particles, $c$ is the concentration of particles, $H$ is the applied magnetic field, $k_{B}$ is the Boltzmann constant, and $T$ is the particle temperature.

A time-independent magnetic field, called a selection field and generated by $I_{D C}$, is applied to obtain an FFP for locating the scanning points, as shown in Fig. 1(b). The magnetic field vanishes at the FFP and its magnitude increases as the distance from the FFP increases. The selection field effectively saturates all nanoparticles outside of the FFP, while the target particles in the FFP and a small region near the FFP are unsaturated. One or more oscillating magnetic fields, called the drive field and generated by $I_{A C}$, are employed to detect the mass of nanoparticles within the FFP, as shown in Fig. 1(c). Movement of the FFP can be achieved by superposition of the 
homogeneous drive field and the selection field (Fig. 1d). The drive field must have sufficiently high frequency to magnetize the nanoparticles at the FFP and generate harmonics that can be received by a receiver coil. The strong signal excluding particles can be isolated by a band-stop filter. The filtered signals indicate the concentration of nanoparticles. The drive field has two functions: generating the particle signal by oscillating magnetization of particles and moving the FFP. Thus, the drive fields should generate sufficient amplitudes to cover the field of view (FOV). Finally, by combining the concentration information with the acquired signal, the nanoparticle spatial distribution can be reconstructed into an image.

It is assumed that the sample is in a 1D shape along the $x$-axis and that the concentration of particles is $c(x)$ along the sample. The MPI only scans the sample in the $x$-direction. For the original 1D MPI concept, first, the target is exposed to a selection field, the magnetic field strength gradient of the selection field along the $x$-axis is $G_{x}$, and a single frequency sinusoidal drive field $H_{D}(t)$ is applied. The signal generated by the oscillating magnetized particles can be described as:

$$
u_{P}(t)=-p_{R} \dot{H}_{D}(t)\left(c^{*} \tilde{m}\right)\left[-G_{x}^{-1} H_{D}(t)\right]
$$

where $H_{D}(t)$ is the drive field used for FFP movement and signal generation, and $p_{R}$ denotes the receiver coil sensitivity along the $x$-axis, which contains all geometrical parameters of the coil. $\tilde{m}(x)=\mu_{0} \dot{\bar{m}}\left(G_{x} x\right)$, where $\dot{\bar{m}}$ denotes the derivative of the mean magnetic moment. The operator * is the convolution of two functions.

\section{B. Signal produced by core-compatible MPI}

For the proposed MPI scheme, the selection field is identical to that in the original MPI scheme and the selection field is generated by $I_{D C}$, as shown in Fig. 1(b). However, the sinusoidal drive field signal in original MPI is separated into two sinusoidal fields of different frequencies which are perpendicular to each other for $x$ and $z$-axis. The first field is a low-frequency drive field with a high amplitude to move only the FFP along the $x$-axis, generated by $I_{A C}$ (see Fig. 1d), and the other is a high-frequency excitation field with a low amplitude, aiming to oscillate the magnetization of the particles and then to generate the signal (Fig. 1e). If the MNPs are saturated and magnetized by the strong field, the excitation field cannot change the magnetization status of these MNPs due to its weak magnitude, and these MNPs also cannot generate any signal. Therefore, if the MNPs are only located at or near the FFP, the excitation field can generate a signal with an existing selection field. Consequently, the total field in the proposed MPI (similar to the drive field in original MPI) is separately given by:

$$
H_{D}(t)=A_{D} \cos \left(2 \pi f_{D} t\right)+A_{E} \cos \left(2 \pi f_{E} t\right)
$$

where $A_{D}$ and $A_{E}$ are the amplitudes of the low-frequency drive field and the excitation field, respectively, and $f_{D}$ and $f_{E}$ are each field frequency. We assume that $A_{E}<<A_{D}$ and $f_{E}>>f_{D}$. Then, since the magnitude of the total field is given by the low-frequency drive field, the total field and its derivative from (4) can be represented as follows:

$$
\begin{gathered}
H_{D}(t) \approx A_{D} \cos \left(2 \pi f_{D} t\right) \\
\dot{H}_{D}(t)=-2 \pi f_{D} A_{D} \sin \left(2 \pi f_{D} t\right)-2 \pi f_{E} A_{E} \sin \left(2 \pi f_{E} t\right)
\end{gathered}
$$

From (3), the voltage induced by the particles can be rewritten as:

$$
u_{P}(t) \approx u_{P L}(t)+u_{P H}(t)
$$

where,

$$
\begin{aligned}
u_{P L}(t)=2 & \pi p_{R} A_{D} f_{D} \sin \left(2 \pi f_{D} t\right) \\
& \cdot\left(c^{*} \tilde{m}\right)\left[-G_{x}^{-1} A_{D} \sin \left(2 \pi f_{D} t\right)\right] \\
u_{P H}(t)=2 & \pi p_{R} A_{E} f_{E} \sin \left(2 \pi f_{E} t\right) \\
& \cdot\left(c^{*} \tilde{m}\right)\left[-G_{x}^{-1} A_{D} \sin \left(2 \pi f_{D} t\right)\right]
\end{aligned}
$$

$u_{P L}(t)$ is the low-frequency component of the particle signal caused by the FFP movement and $u_{P H}(t)$ is the high-frequency component of the particle signal, caused by the excitation frequency $f_{E}, p_{R}$ denotes the receiver coil sensitivity. By applying the coordinate transform introduced in [18], we obtain:

$$
\begin{gathered}
x_{F F P}(t)=-G_{x}^{-1} A_{D} \cos \left(2 \pi f_{D} t\right) \\
\dot{x}_{F F P}(t)=2 \pi G_{x}^{-1} f_{D} A_{D} \sin \left(2 \pi f_{D} t\right)
\end{gathered}
$$

where $x_{F F P}$ and $\dot{x}_{F F P}$ are the position and its derivative of the FFP, respectively. Equation (10) indicates that the FFP position depends on the amplitude of the low-frequency drive field and the gradient of the selection field. The time signals (7), (8), and (9) can be mapped onto a spatial interval and described by an ordinary convolution:

$$
u_{P}\left[x_{F F P}(t)\right] \approx u_{P L}\left[x_{F F P}(t)\right]+u_{P H}\left[x_{F F P}(t)\right]
$$

Equations (8) and (9) can be converted into

$$
\begin{gathered}
u_{P L}\left[x_{F F P}(t)\right]=p_{R} G_{x}\left(c^{*} \tilde{m}\right)\left[x_{F F P}(t)\right] \dot{x}_{F F P}(t) \\
u_{P H}\left[x_{F F P}(t)\right]=2 \pi p_{R} A_{E} f_{E}\left(c^{*} \tilde{m}\right)\left[x_{F F P}(t)\right] \sin \left(2 \pi f_{E} t\right)
\end{gathered}
$$

Equation (13) indicates that the low-frequency signal is the product of the speed of the FFP, expressed by $\dot{x}_{F F P}$, and the concentration of particles at the FFP. Equation (14) indicates that the high-frequency signal is a sinusoidal voltage under the frequency $f_{E}$, with an amplitude proportional to the particle concentration at the FFP and the amplitude and frequency of the excitation field. Equation (14) also represents the suggested AM MPI requires the amplitude of the received signal to reconstruct the MPI images. To only receive $u_{\mathrm{PH}}$, the excitation and receiver coils should be located along the $z$-axis. 


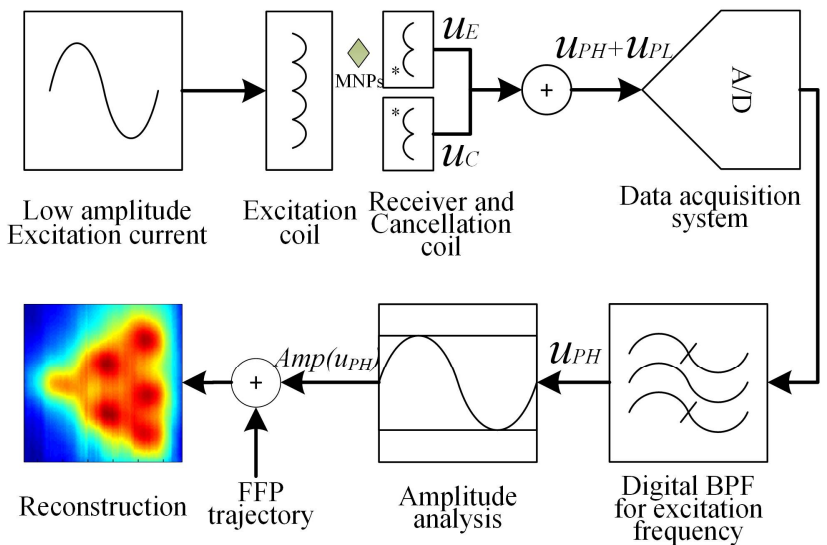

Fig. 3. Signal flow diagram of the realized set-up.

\section{Signal processing and reconstruction of the core-compatible MPI}

Both $u_{P L}(t)$ and $u_{P H}(t)$ can be used for image reconstruction of particles. For $u_{P L}(t)$, we can utilize the position of the FFP, the speed of the FFP, and the magnitude of the signal for reconstruction using the X-space method [19]. However, if we use $u_{P L}(t)$, the boundary effect for zero FFP speed near the boundary will lead to a very low signal-to-noise ratio (SNR). Furthermore, since the speed of the FFP is unmeasurable when extending to $3 \mathrm{D}$, the $\mathrm{X}$-space method will increase the difficulty of reconstruction. Compared to $u_{P L}(t), u_{P H}(t)$ requires only the amplitude and the FFP position to reconstruct the image and is independent of the FFP speed. Moreover, the higher excitation frequency will improve the signal-noise-ratio [20]. Hence, $u_{P H}(t)$ is used for image reconstruction.

A signal processing procedure for determining the particle concentration is described in Fig. 3. When the excitation coil in MPI is supplied by a low-current circuit, the high-frequency and low-amplitude excitation field is induced for magnetization of the MNPs. Then, the magnetized particles generate the signal, which will be received by a receiver coil. The excitation field coupled to the receiver coil also induces a signal with an excitation frequency. This signal exists even when the receiver coil has no MNPs inside, called the excitation signal $u_{E}^{\prime}(t)$, which is measured from the receiver coil when MNPs are not located inside the receiver coil and should be eliminated by a cancellation coil [21]. The cancellation coil and the excitation coil, with the same geometric parameters (such as coil sizes, wire specifications, and number of turns) but wound in opposite directions, are connected in series and placed in the excitation field. The excitation field will induce AC voltages in both coils $\left(u_{E}^{\prime}(t)\right.$ in the receiver coil and $u_{C}(t)$ in the cancellation coil) due to Faraday's law. Because of their identical geometric parameters, the induced voltages in the two coils have the same amplitudes but opposite directions ( or $u_{E}^{\prime}(t) \approx-u_{\mathrm{C}}(t)$ ), resulting in cancellation of both voltage phases; thus, the total voltage of the receiver coil and the cancellation coil is reduced to approximately $0 \mathrm{~V}$. When the MNPs are located inside the receiver coil, the excitation signal becomes $u_{\mathrm{E}}(t)$ and it includes the particle signal as follows:

$$
u_{E}(t)=u_{E}^{\prime}(t)+u_{p}(t)
$$

Then, the particle signal $u_{\mathrm{P}}(t)$ can be measured by combining the signals of the receiver coil and the cancellation coil as follows:

$$
u_{P}(t) \approx u_{E}(t)+u_{C}(t)
$$

The particle signal can be digitized using a DAQ acquisition system; then, the signal can be filtered using a band-pass filter (BPF), which determines the high-frequency components of the signal $\left(u_{P H}\right)$ with a narrow-band frequency. Therefore, a narrow-band receiver coil for the excitation frequency is sufficient for acquiring the particle signal. According to equation (14), the amplitude of the filtered signal contains information on particle concentration at the FFP. The amplitude of the signal is given by

$$
A m p\left[u_{P H}\left(x_{F F P}\right)\right]=2 \pi p_{R} A_{E} f_{E}\left(c^{*} \tilde{m}\right)\left[x_{F F P}\right]
$$

For a MPI system, $A_{E}, f_{E}$, and $p_{R}$ are constants and the amplitude of the high-frequency signal component is only linked to MNP density. Thus, this signal combined to the present trajectory of the FFP can be easily reconstructed. Once we measure the MPI signal, we can generate the AM MPI image by gridding of the received signal to the instantaneous position of the FFP. For reconstruction, the resulting image can be obtained as

$$
\operatorname{IMG}(x)=\operatorname{Amp}\left[u_{P H}(x)\right]
$$

where $\mathrm{x}$ is the FFP position. Equation (18) represents that the value of every pixel in the AM MPI image is the amplitude of the high-frequency signal when the FFP arrives.

\section{IRON CORES IN MPI}

The original MPI should not include any components made of soft magnetic materials with a nonlinear magnetization curve, as the soft magnetic material will generate harmonic noise in the receiver coil, screening the particle signal. As a result, the original MPI method will not be available with the core-coil system. However, the soft iron cores can be applied to a navigation system for MNPs based on the proposed AM MPI scheme. This chapter will discuss in details how the proposed MPI scheme is available.

\section{A. Simulation and experimental setup for coil-core structure}

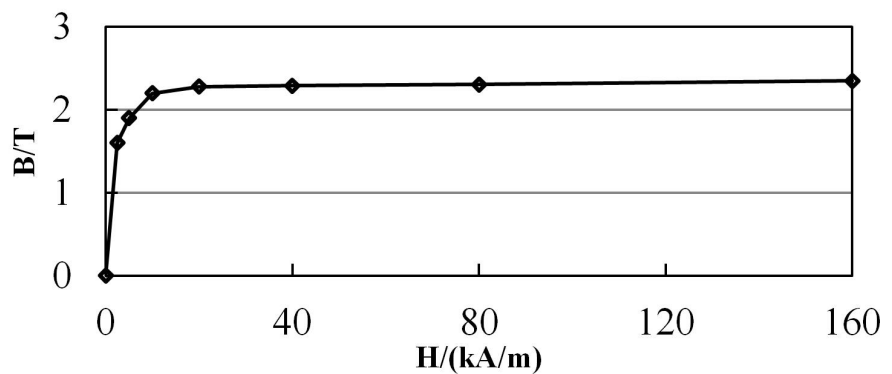

Fig. 4. B-H curve of VACOFLUX 50. 


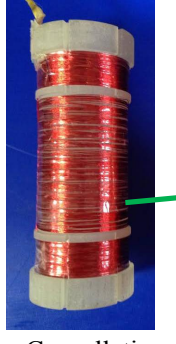

Cancellation

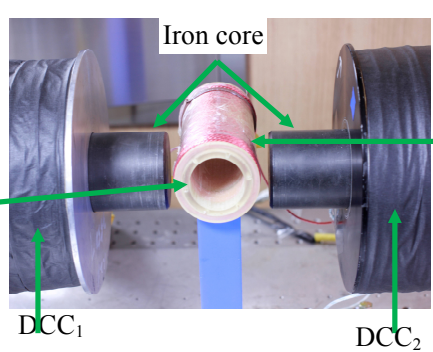

and receiver coil

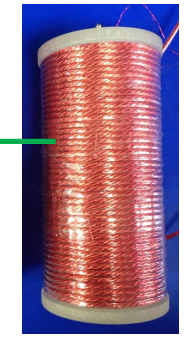

Low-amplitude excitation coil
Fig. 5. Experimental set-up for hybrid electromagnetic actuation and imaging of MNPs with an iron core.

The iron core in our electromagnetic actuation system is composed of soft magnetic alloys (VACOFLUX 50, Vacuumschmelze) and its B-H curve is shown in Fig. 4. For comparison and objective analysis of the electromagnetic systems with the iron-core coil and with the air-core coil, the size of the workspaces and the gap between DCCs were set to the same values, as shown in Fig. 2. The coil parameters are shown in Table 1 for both simulation and experiments. The core is located inside an inner coil of the DCCs, and each core has a cylindrical shape with a length of $19.5 \mathrm{~cm}$ and a diameter of 6 $\mathrm{cm}$. The experimental set-up for hybrid electromagnetic actuation and imaging of MNPs was devised to verify the availability of the coil-core topology design, as shown in Fig. 5. The two actuation coils with iron cores are powered by two DC power supplies (AMETEK SGA 600/17, $10 \mathrm{~kW}$ ), and a sinusoidal signal with a high frequency generated by a function generator (KEYSIGHT 33500B Series) is amplified by a power amplifier (AE TECHRON 7224), with the resultant current being fed to the excitation coil.

Table 1 Coil specifications

\begin{tabular}{l|cclll}
\hline & Turns & $\begin{array}{l}\text { Inner } \\
\text { diameter }\end{array}$ & $\begin{array}{l}\text { Outer } \\
\text { diameter }\end{array}$ & $\begin{array}{l}\text { Coil } \\
\text { length }\end{array}$ & Wire \\
\hline DCC & $\begin{array}{l}5000 \\
\text { each }\end{array}$ & $7 \mathrm{~cm}$ & $19 \mathrm{~cm}$ & $7 \mathrm{~cm}$ & $\begin{array}{l}1 \mathrm{~mm} \\
\text { copper } \\
\text { wire }\end{array}$ \\
$\begin{array}{l}\text { Receiver } \\
\text { coil }\end{array}$ & 400 & $4.2 \mathrm{~cm}$ & $\begin{array}{l}\text { Single } \\
\text { layer }\end{array}$ & $6 \mathrm{~cm}$ & Litz wire \\
$\begin{array}{l}\text { Cancellation } \\
\text { coil }\end{array}$ & $\begin{array}{l}200 \\
\text { each }\end{array}$ & $4.2 \mathrm{~cm}$ & $\begin{array}{l}\text { Single } \\
\text { layer }\end{array}$ & $1.5 \mathrm{~cm}$ & Litz wire \\
$\begin{array}{l}\text { Excitation } \\
\text { coil }\end{array}$ & 44 & $5 \mathrm{~cm}$ & $\begin{array}{l}\text { Single } \\
\text { layer }\end{array}$ & $11 \mathrm{~cm}$ & Litz wire \\
\hline
\end{tabular}

\section{B. Selection field effects with the coil-core structure}

The magnetic field gradients in the air-core system and the iron-core system are compared in Fig. 6. The magnitude of the magnetic field gradient in the iron-core system at $3 \mathrm{~A}$ is almost equal to the magnitude of the magnetic field gradient in the air-core system at $7 \mathrm{~A}$. If the iron-core system is loaded at $7 \mathrm{~A}$, the magnitude of the magnetic field gradient is almost three times that of the air-core system. One hypothesis is that the selection field, $G_{x}$, is linear within the workspace and constant in equation (3). When utilizing soft magnetic components in the proposed MPI system, $G_{x}$ can also be regarded as a constant due to the linear selection field within the workspace.

\section{Drive field effects with the coil- core structure}

The sinusoidal currents loaded in the air-core coils can generate orthogonal sinusoidal fields for the FFP to scan as a sinusoid curve or a Lissajous trajectory, depending on the linearity of the relationship between the current input and the field induction of the air-core coil. However, for the iron-core coil, the input current and field output will exhibit a complex relationship due to the nonlinear magnetization process of the core. Simulations and experiments were carried out to investigate this nonlinear relationship, and the results of the magnetic field of the iron-core coil with respect to the current are shown in Fig. 7.

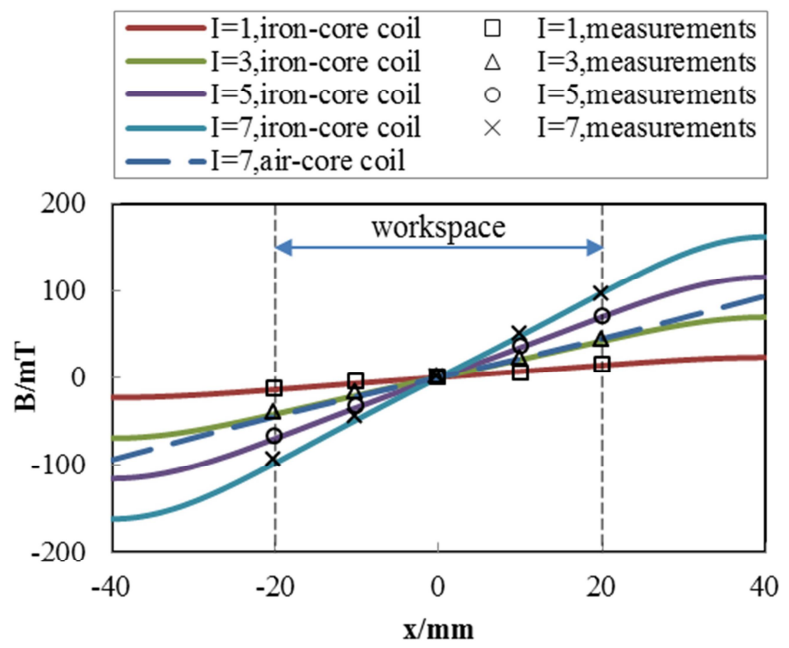

Fig. 6. The selection field generated by two DCCs with its axial axis located along the $x$-axis. Both sides of the coil load an equal and opposite current. Simulation results and measurements are marked by solid lines and scattered points, respectively. The field is highly linear in the workspace for iron-core and air-core coils. The slope of the curve indicates the gradient. The field gradients of the iron-core coils are much greater than those of the air-core coils. When a 3 A current is loaded onto the iron-core coils, the field gradient is comparable to that of the air-core coils with a 7 A current.

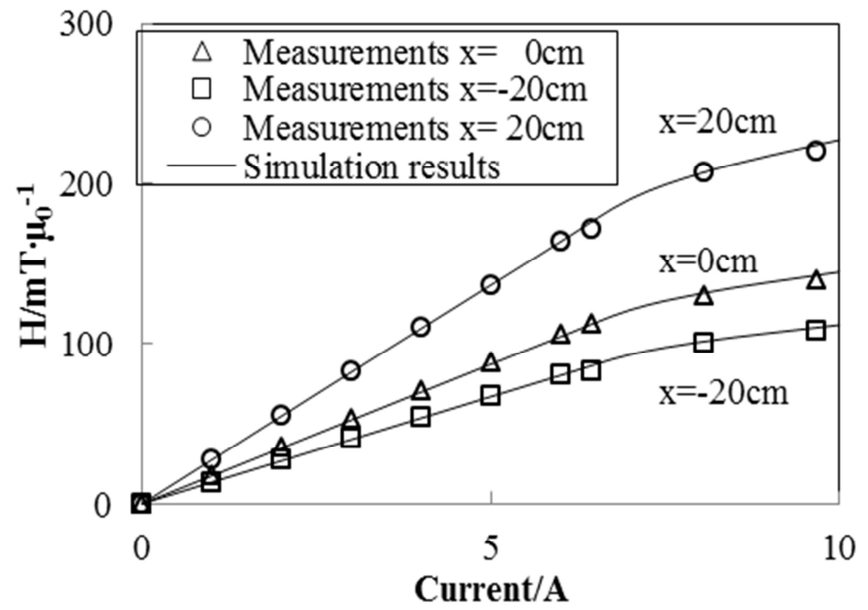

Fig. 7. Relationship of the magnetic field strength vs. current in the iron-core system; the study points are at the center and the two edges of the workspace. The magnetic field strength is proportional to the input current. 
An inflection point occurs on the curve at a current of $7 \mathrm{~A}$. The current and the magnetic field of the iron-core coil have a linear relation for currents smaller than $7 \mathrm{~A}$, while the input and output have a nonlinear relation for currents larger than $7 \mathrm{~A}$. This phenomenon is due to the non-uniform magnetization of the core. For currents lower than the inflection current, the whole core will maintain an unsaturation condition, while part of the core will be saturated for currents higher than the inflection current, and reflect the nonlinearity of the outer field. Therefore, it is recommended that the linear condition of the iron-core coil, for currents lower than $7 \mathrm{~A}$, be utilized in the current system. The hypothesis of equation (4) is that the magnetic field amplitude $A_{D}$ and the input current amplitude $I_{A C}$ have a linear relationship:

$$
A_{D}=p_{D} I_{A C}
$$

where $p_{D}$ is the sensitivity of the DCC and is independent of input current. If the current is lower than $7 \mathrm{~A}$, the received signal in equation (14) can be directly applied in MPI with iron cores.
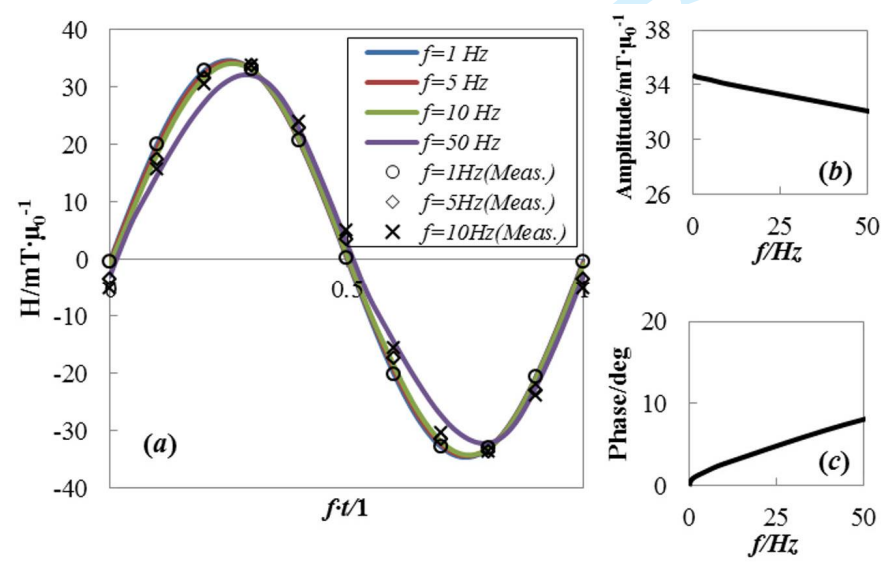

Fig. 8. Simulation and experimental results of magnetic field strength vs. frequency. The sinusoidal currents are loaded onto the iron-core coils and these currents have the same amplitude but different frequency. (a) Magnetic field strength in different phases. (b) Amplitude decreases with respect to frequency. (c) Phase delay increases with respect to frequency.

Another effect on the trajectory is the low-frequency eddy current in the core, induced by the low-frequency drive field. While using currents with the same amplitude but different frequency to achieve FFP scanning, the effective field for steering the FFP is dependent on the frequency, as shown in Fig. 8. Fig. 8(a) shows the magnitudes of the field in the time domain with different frequencies, and Fig. 8(b) shows that the amplitude of the magnetic field strength decreases with respect to the frequency. The amplitude of the low-frequency drive field in the time domain only affects the size of the workspace. If a higher low-frequency drive field frequency is needed, the amplitude of the currents should be increased slightly to maintain the size of the workspace. Furthermore, phase delays under different frequencies are shown in Fig. 8(c). If the frequency is less than $10 \mathrm{~Hz}$, the phase delay of the magnetic field caused by the iron cores is less than $0.05 \mathrm{rad}$ (about $2 \%$ ) therefore, the effects of the phase delay and the amplitude of the low-frequency drive field are insignificant and can be neglected in practice when the current frequency for the low-frequency drive field is lower than $10 \mathrm{~Hz}$ in the iron-core coil system.

\section{Excitation field effects with the coil-core structure}

When the iron cores are exposed simultaneously to a strong selection field and a sufficient small-amplitude high-frequency excitation field during MPI mode, the iron cores will respond approximately as the sum of the sinusoidal magnetization and the static magnetization. Moreover, even though an eddy current on the skin of the core can be generated when exposed to the high-frequency excitation field, the noise induced by the iron cores can be considered a part of the empty signal and removed by using the cancellation coil.

\section{RESUlts OF 1D NAVIGATION OF MNPS AND 2D IMAGING}

\section{A. Experimental set-up for navigation}

In this chapter, we aim to implement the navigation system for MNPs using the iron-core electromagnetic system and iron-core compatible MPI scheme. Fig. 9 shows the hardware connections for the proposed navigation system. The nanoparticles used to verify the 1D and 2D MPI system are 45 to $65 \mathrm{~nm}$ in diameter with a core size of 5 to $6 \mathrm{~nm}$ (Resovist, Meito Sangyo Co., Ltd.). The Fe content and the magnetic susceptibility of the undiluted sample were about $58.6 \mathrm{mg} / \mathrm{mL}$ and $0.035 \mathrm{erg} \cdot$ gauss $^{\prime} \mathrm{g}^{-1}$, respectively, and saturation magneti zation values were $340 \pm 10 \mathrm{kA} / \mathrm{m}$ [22]. The length of the sample tank was $20 \mathrm{~mm}$, and it was filled with $50 \mu \mathrm{L}$ of undiluted MNP suspension and $570 \mu \mathrm{L}$ of oil. The MNP suspension is water-based and will not mix with the oil; therefore, the MNPs in the oil form spherical droplets under the magnetic field, as shown in Fig. 11. The navigation system can steer the droplet along the $x$-axis (sample tank) and monitor the MNPs' positions in the real-time MPI images. To verify the real-time monitoring and steering results, the positions of the droplet are also observed with an optical camera.

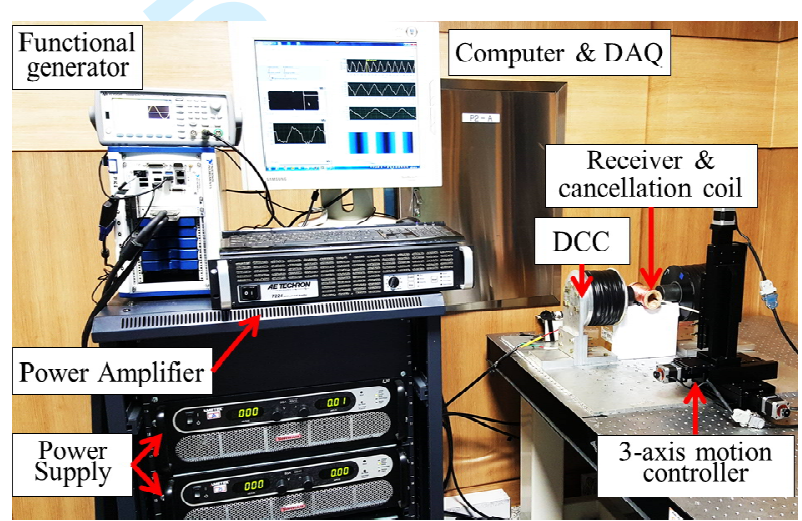

Fig. 9. Two power supplies, which are controlled separately by PC LabVIEW controller, supply currents to DCCs for generating FFP, moving FFP, and generating a magnetic gradient field to steer MNPs. A current generated by a function generator and amplified by a power amplifier is supplied to an excitation coil with low amplitude and high frequency. DAQ system is used to collect signals from a receiver coil and a cancellation coil.

\section{B. 1D particle navigation}

The MNPs were placed along the FFP scanning trajectory so that their concentration distribution could be monitored using 


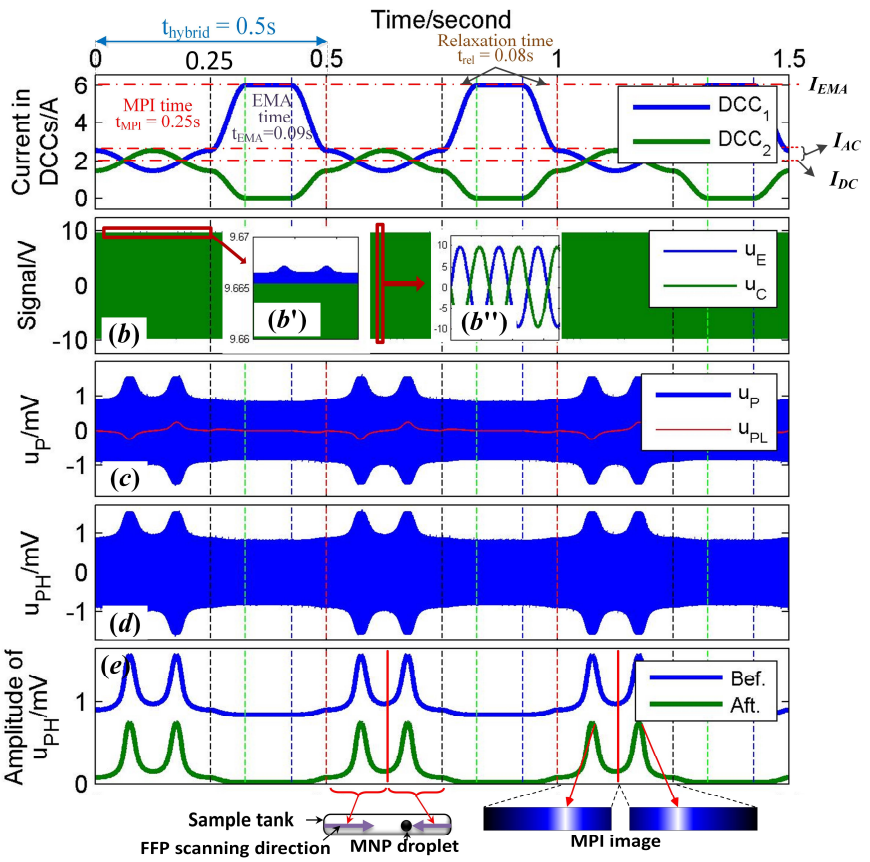

Fig. 10. 1D navigation results for MNPs with seting parameters as follow: $t_{\text {hybrid }}$ $=0.5 \mathrm{sec}$; for MPI period: $t_{\mathrm{MPI}}=0.25 \mathrm{sec}, I_{\mathrm{DC}}=2 \mathrm{~A}, I_{\mathrm{AC}}=0.88 \mathrm{~A}$; for relaxation period: $t_{\mathrm{rel}}=0.08 \mathrm{sec}$; for EMA period: $t_{\mathrm{EMA}}=0.09 \mathrm{sec}, I_{\mathrm{EMA}}=6 \mathrm{~A}$. (a) shows the currents waveform in the DCCs. Relaxation times are also included to prevent voltage surge. (b) compares $u_{E}$ and $u_{C}$. There is invisible difference between $u_{E}$ and $u_{C}$ in full scale. (b') The amplitudes of $u_{E}$ and $u_{C}$ have slight differences, which are caused by MNPs. (b") $u_{E}$ and $u_{C}$ have the equal frequency with a phase difference of $180 \mathrm{deg}$. (c) shows the low frequency component $u_{\mathrm{PL}}(\mathrm{t})$ and the total voltage $u_{p}(\mathrm{t})$ induced by particles. Because the directions of the excitation field and the low-frequecy drive field are perpendicular to each other, $u_{p}(t)$ has little effects for $u_{\mathrm{PL}}(t)$. (d) shows the high frequency component $u_{\mathrm{PH}}(\mathrm{t})$, whose amplitude is shown in (e). (e) The empty signal cannot be reduced to exactly $0 \mathrm{~V}$ by the cancellation coil due to manufacturing errors. But since the amplitude of the empty signal is consistent, the amplitude of the empty signal can be subtracted from the amplitude of the signal. The blue line shows the amplitude before the correction; the green line shows the amplitude after the correction. Only signal segments during MPI mode are useful. The MPI time includes two full MPI scanning periods in different directions. Each scan includes full MNP distribution information.

MPI. Simultaneously, EMA mode can steer the MNPs in the same direction inside the tube. The MNP concentration was estimated from the amplitude of the received signal. When a magnetic field was applied, the MNPs were aggregated into small droplets. In this experiment, the initial position of the droplet is on the left end of the tank, and a constant current was applied to the right coil to guide the droplet to the right using EMA mode. The droplet is monitored simultaneously using MPI mode. Guidance was terminated when the MNP droplet reached the right side of the tank. The resultant signals are shown in Fig. 10, and snapshots taken during the experiments of MPI images and locations are shown in Fig. 11. Droplet positions monitored by real-time MPI are shown in Fig. 12.

The maximum values in the MPI image are thought to indicate the center of the MNP droplets. The grayscale images are reconstructed for MPI images and illustrate the MNP concentrations; black indicates an MNP concentration of zero while white indicates the highest MNP concentrations along the sample tank. The experimental results (Fig. 11) verified that the navigation system can monitor the positions of MNPs and feed

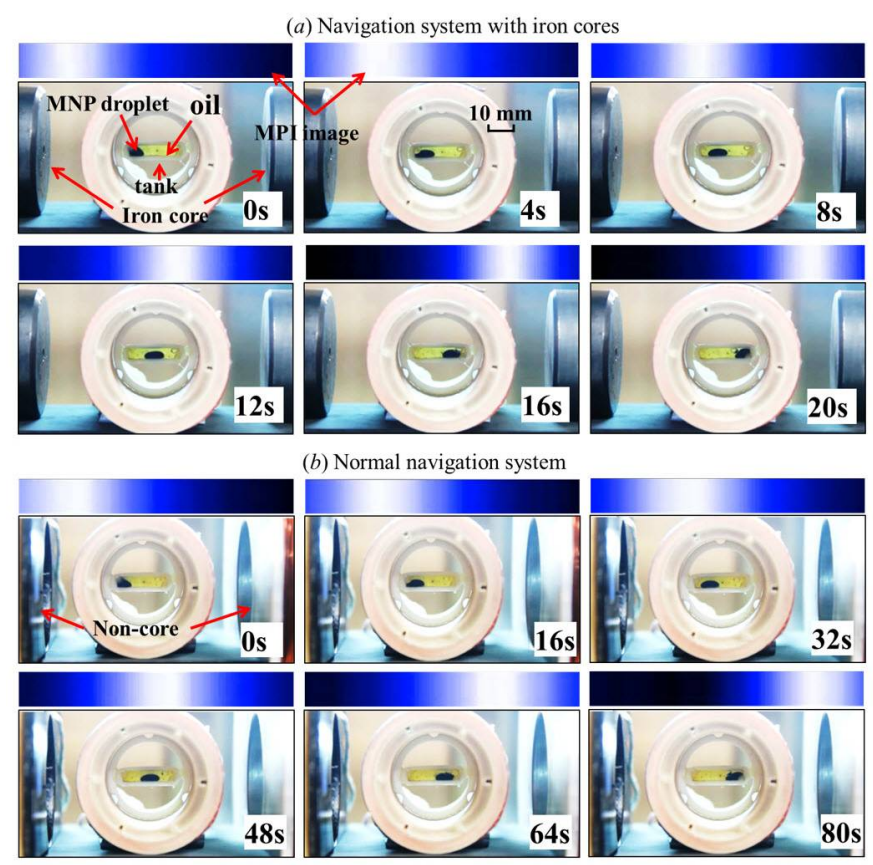

Fig. 11. Results of experiments using Resovist MNPs manipulated by the navigation system. During the EMA periods, the force was towards the right side of the sample tank $\left[I_{E M A}=6 \mathrm{~A}(3.12 \mathrm{~T} / \mathrm{m})\right]$. The camera images and the MPI images (above the camera images) show the position of the particle droplet. The white regions indicate a higher MNP concentration. A magnetic field gradient of $1.04 \mathrm{~T} / \mathrm{m}$, induced by $I_{D C}=2 \mathrm{~A}$, was used in MPI mode. $I_{A C}$ was set to 0.88 A to incorporate a workspace of $4 \mathrm{~cm}$. The excitation field was $200 \mu \mathrm{T}$ and $40 \mathrm{kHz}$. (a) Guidance by a navigation system with iron cores. (b) Guidance by a normal navigation system.

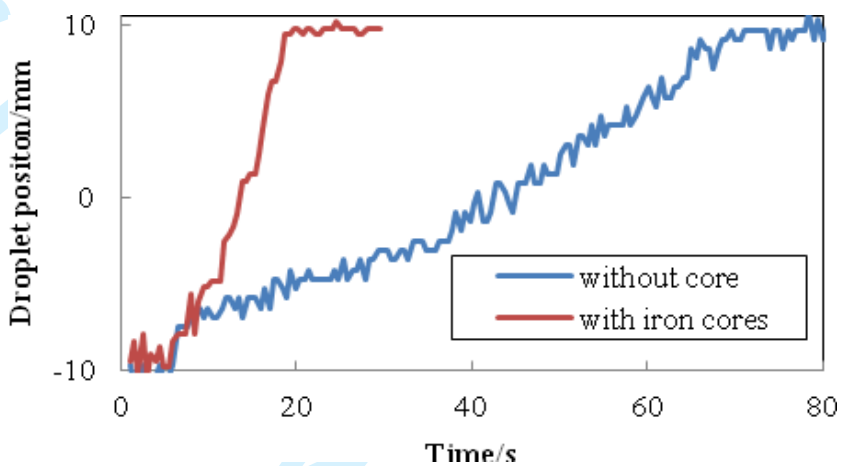

Fig. 12. Positions of the MNP droplets during navigation with and without cores.

Table 2. Guidance times of MNPs with and without cores inside a sample tank

\begin{tabular}{ccccc}
\hline \multirow{2}{*}{$I_{A C}$} & $I_{D C}$ & $I_{E M A}$ & $\begin{array}{c}\text { with } \\
\text { cores }\end{array}$ & $\begin{array}{c}\text { without } \\
\text { cores }\end{array}$ \\
\hline \multirow{3}{*}{$0.88 \mathrm{~A}$} & & $6 \mathrm{~A}$ & $20 \mathrm{sec}$ & $80 \mathrm{sec}$ \\
& $2 \mathrm{~A}$ & $5 \mathrm{~A}$ & $34 \mathrm{sec}$ & $105 \mathrm{sec}$ \\
& $4 \mathrm{~A}$ & $48 \mathrm{sec}$ & $140 \mathrm{sec}$ \\
& $3 \mathrm{~A}$ & $82 \mathrm{sec}$ & $190 \mathrm{sec}$ \\
\hline \multirow{3}{*}{$1.76 \mathrm{~A}$} & $6 \mathrm{~A}$ & $26 \mathrm{sec}$ & $66 \mathrm{sec}$ \\
& & $5 \mathrm{~A}$ & $33 \mathrm{sec}$ & $81 \mathrm{sec}$ \\
& & $4 \mathrm{~A}$ & $40 \mathrm{sec}$ & $100 \mathrm{sec}$ \\
& & $3 \mathrm{~A}$ & $68 \mathrm{sec}$ & $150 \mathrm{sec}$ \\
\hline
\end{tabular}

particle concentration information back to EMA mode; then, the system guides MNPs to expected locations. Our 1D MNP navigation system had a $2 \mathrm{~Hz}$ system refresh frequency (repetition frequency), and the MPI image frame and control field were updated every $0.5 \mathrm{~s}$, which is an appropriate 
bandwidth for navigating capillaries in the brain, where the velocity of blood is approximately $1 \mathrm{~mm} / \mathrm{s}$ [23].

The same current conditions were applied in the navigation systems with and without iron cores to observe the steering force effects of the iron core using the conditions of Fig. 2. The durations for guiding the MNP droplets from the left to the right of the tank are given in Table 2. $I_{E M A}$ is increased to speed up the MNPs during EMA modes, while $I_{A C}$ was adjusted to maintain the size of workspace at $4 \mathrm{~cm}$. The results show that by installing the soft magnetic cores, the guidance time can be significantly reduced and that real-time MPI imaging is still available by utilizing the proposed AM MPI scheme with higher imaging resolution.

To extend the $1 \mathrm{D}$ navigation system to a $3 \mathrm{D}$ system, two sets of iron-core coils, which can induce fields orthogonal to each other, should be included in the proposed system. These coils can act as the actuation coils in the EMA to steer MNPs in a 3D target region and the drive coils in the MPI mode to monitor the MNP distribution. By choosing currents of appropriate frequency in these two sets of coils, a 3D Lissajous trajectory can be achieved to cover a 3D workspace, and the received signal can then be reconstructed to $3 \mathrm{D}$ MPI images. We estimated that, if we adopted $24 \mathrm{~Hz}$ and $20 \mathrm{~Hz}$ sinusoidal currents in these two sets of coils during the MPI periods, the navigation system would retain a $2 \mathrm{~Hz}$ system refresh frequency. When the frequency of the drive field is higher than $10 \mathrm{~Hz}$, phase delays can affect the receiver signal; to remove these phase delay effects, the measured signal can be deconvolved with the estimated time constant, which reveals the underlying mirror symmetry [24]. Through this compensation, an exact image of MNPs in the 3D system can be obtained. All of these conditions are possible with our current power supply with a current slew rate of $153 \mathrm{~A} / \mathrm{s}$.

\section{C. $2 D$ imaging}

Fig. 13 shows 2D images with different gradients. For this experiment, EMA mode was deactivated and the navigation system had a 1D MPI functionality: only MNPs along the DCC $(x)$ axis can be scanned (Fig. 2). The MPI scanning frequency in our system was $10 \mathrm{~Hz}$, and a three-axis motion controller (st1, Korea) was used to move a $2 \mathrm{D}$ sample (see Fig. 13) at a constant velocity through the DCC axis. The movement direction was along the $\mathrm{z}$-axis of the receiver coil, as shown in Fig. 2. In the experiments, the velocity was set to $2 \mathrm{~mm} / \mathrm{s}$, and the sampling time for each image was 15 seconds. When the excitation fields for different scans were maintained at $200 \mu \mathrm{T}$ and $40 \mathrm{kHz}$, the images became clear when increasing the $\mathrm{I}_{\mathrm{DC}}$.

The 2D MPI images created from two samples are shown in Fig. 13. In each figure, (a) shows the samples, (b)-(e) show the images from iron-core MPI, and (f)-(i) show the images from MPI without cores. Sample (1) shows MPI images of six Resovist MNP droplets, with each droplet including $10 \mu \mathrm{L}$ of Resovist MNPs. The gradient field had a positive effect on MPI image resolution such that as the gradient increased each droplet became clearer. Sample (2) in Fig. 13 demonstrates images of a Y-shaped channel, which simulates bifurcation of a small blood vessel. This MPI image can be combined with

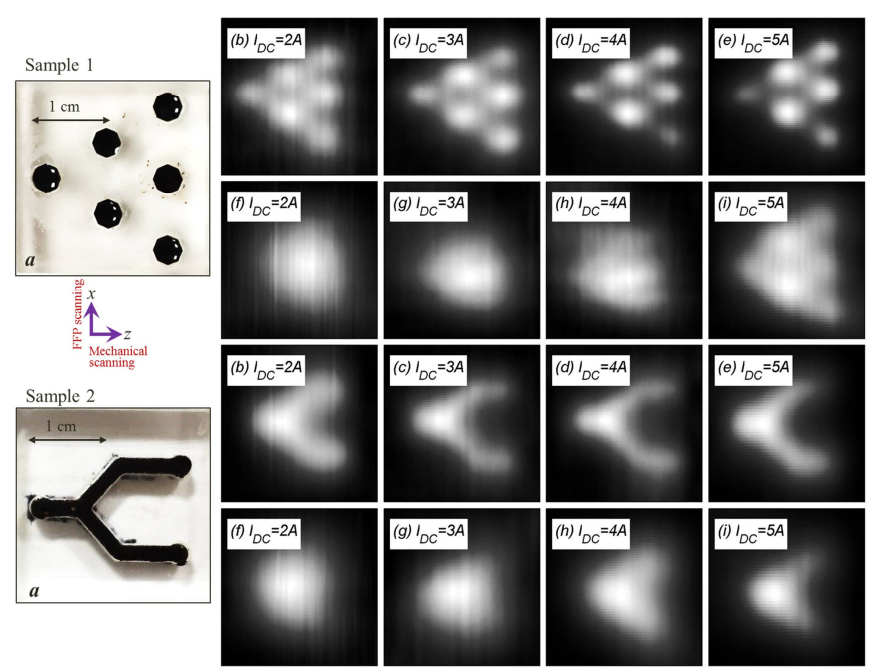

Fig. 13. Reconstructed images of the object for two samples: sample (1): MPI images of six Resovist MNPs droplets, each droplet includes $10 \mu \mathrm{L}$ of Resovist MNPs; sample (2): Images of a Y-shaped channel with a diameter of $1 \mathrm{~mm}$. (a) shows the sample, (b)-(e) results are generated from the iron-core MPI system, and (f)-(i) results are created from the MPI system without a core. The results under the same current conditions in two different systems are compared. For the system without a core, when the current was too low, the gradient was not sufficient to generate clear images (f, g, and h). For different $I_{D C}$, the excitation fields for different scans were maintained at $200 \mu \mathrm{T}$ and $40 \mathrm{kHz}$. For $I_{D C}=5 \mathrm{~A}$, the resultant gradient field $G_{x}$ was $2.6 \mathrm{~T} / \mathrm{m}$. The sampling times were 15 seconds.

EMA periods for MNP steering. The MPI images generated by the MPI systems with and without iron cores clearly show that MPI images with cores were clearer. Even though the best resolution with a Resovist particle with a 5-nm core size is larger than $4 \mathrm{~mm}$ with the proposed navigation system, millimeter-scale resolution can be achieved using bigger MNPs, or increasing magnetic gradient field [9].

These results show that the proposed MPI method can provide three major benefits: improved resolution without increasing the amplitude of the high-frequency magnetic field; maintaining the availability of MPI with iron cores; and use of less electrical energy to generate the same gradient compared to an air-core coil system. These features are all beneficial for extending the workspace of MPI using a low-cost method.

\section{CONCLUSION}

A navigation system with iron cores is proposed that is cost-efficient, compact, and optimized for precise targeting of the MNPs by implementing real-time actuation and monitoring. The design is suitable for a low-blood-speed application. These novel techniques can be adapted for a variety of applications, particularly direct delivery of nanomedicines. The proposed system can allow a user to manipulate MNPs in response to real-time MPI images. By mixing two frequency fields, the proposed AM MPI scheme makes soft magnetic components accessible to an MPI system. Soft magnetic components can enhance the magnetic field gradient and strength, and provide a higher steering force for MNPs and enhanced MPI resolution. The electromagnetic system with iron-cores can save up to $67 \%$ of the required current compared to a system without cores; thus, the quantity of heat generated is only one-ninth of that of the non-core system. In addition to the higher gradient field due 
to the iron cores, the narrow-band receiver coil, the low-amplitude excitation field, the low-frequency drive field, and the current savings make the proposed MPI highly scalable. For in vivo imaging at the human scale, several design improvements and innovations must be introduced to the present system. Real-time implementation of a mouse-sized system and experiments in a vascular network with many bifurcations will be studied in future work.

\section{ACKNOWLEDGMENTS}

This work was supported by the National Research Foundation Korea (NRF) (2012-0009524 and 2014R1A2A1A11053989) and the Scientific Research Foundation of Harbin Institute of Technology at Weihai (HIT(WH)201604).

\section{REFERENCES}

[1] S.C. Mcbain, H.H. Yiu, J. Dobson, "Magnetic nanoparticles for gene and drug delivery," International Journal of Nanomedicine. vol. 3 no. 2, pp. 169-180, 2008.

[2] B. Gleich and J. Weizenecker, "Tomographic imaging using the nonlinear response of magnetic particles," Nature, vol. 435, pp. 1214-1217, 2005.

[3] F. Dilnawaz, S. K. Sahoo, "Therapeutic approaches of magnetic nanoparticles for the central nervous system," Drug Discovery Today, vol. 20, no. 10, pp. 1256-1264, 2015.

[4] M. O. Kim, F. U. Amin, A. K. Hoshiar, T. D. Do, Y. Noh, S. A. Shah, M. S. Khan, J. Yoon, "Osmotin-loaded Magnetic Nanoparticles with an Electromagnetic Guidance for the Treatment of Alzheimer's disease," Nanoscale, online publication on 03 May 2017, DOI:10.1039/C7NR00772H

[5] I. S. M. Khalil, P. Ferreira, R. Eleuterio, C. L. de Korte, and S. Misra, "Magnetic-Based Closed-Loop Control of Paramagnetic Microparticles using Ultrasound Feedback," IEEE International Conference on Robotics and Automation ICRA, 2014, pp. 3807-3812.

[6] I. S. Khalil, J. D. Keuning, L. Abelmann, and S. Misra, "Wireless magnetic-based control of paramagnetic microparticles," 2012 4th IEEE RAS \& EMBS International Conference on Biomedical Robotics and Biomechatronics (BioRob), 2012, pp. 460-466.

[7] S. Martel, O. Felfoul, J. B. Mathieu, A. Chanu, S. Tamaz, M. Mohammadi, M. Mankiewicz, and N. Tabatabaei, "MRI-based medical nanorobotic platform for the control of magnetic nanoparticles and flagellated bacteria for target interventions in human capillaries," International Journal of Robotics Research, vol. 28, no. 9, pp. 1169-1182, 2009.

[8] S. Martel, "Beyond imaging: Macro- and microscale medical robots actuated by clinical MRI scanners," Science Robotics, vol. 2, eaam8119, 2017.

[9] P. W. Goodwill and S. M. Conolly, "The X-Space Formulation of the Magnetic Particle Imaging Process: 1-D Signal, Resolution, Bandwidth, SNR, SAR, and Magnetostimulation," IEEE Transactions on Medical Imaging, vol. 29, pp. 1851-1859, 2010.

[10] E. M. Cherry, P. G. Maxim and J. K. Eaton, "Particle size, magnetic field, and blood velocity effects on particle retention in magnetic drug targeting," Medical Physics, vol. 37, pp. 175-182, 2010. [11] B. Shapiro, S. Kulkarni, A. Nacev, S. Muro, P. Y. Stepanov, and I. N. Weinberg, "Open challenges in magnetic drug targeting," WILEY Interdisciplinary Reviews-Nanomedicine and Nanobiotechnology, vol. 7, pp. 446-457, 2015.

[12] I. N. Weinberg, P. Y. Stepanov, S. T. Fricke, R. Probst, M. Urdaneta, D. Warnow, H. Sanders, S. C. Glidden, A. McMillan, P. M. Starewicz, and J. P. Reilly, "Increasing the oscillation frequency of strong magnetic fields above $101 \mathrm{kHz}$ significantly raises peripheral nerve excitation thresholds," Medical Physics, vol. 39, pp. 2578-2583, 2012.

[13] P. W. Goodwill, G. C. Scott, P. P. Stang, and S. M. Conolly, "Narrowband Magnetic Particle Imaging," IEEE Transactions on Medical Imaging, vol. 28, pp. 1231-1237, 2009.

[14] M. D. Tehrani, M. O. Kim and J. Yoon, "A novel electromagnetic actuation system for magnetic nanoparticle guidance in blood vessels," IEEE Transactions on Magnetics, vol. 50, pp. 1-12, 2014.

[15] M. Latulippe and S. Martel, "Dipole field navigation: Theory and proof of concept," IEEE Transactions on Robotics, vol. 31, pp. 1353-1363, 2015

[16] X. Zhang, T. Le and J. Yoon, "Development of a magnetic nanoparticles guidance system for interleaved actuation and MPI-based monitoring," in Intelligent Robots and Systems (IROS), 2016 IEEE/RSJ International Conference on, 2016, pp. 5279-5284.

[17] A. Mahmood, M. Dadkhah, M. O. Kim, and J. Yoon, "A novel design of an MPI-based guidance system for simultaneous actuation and monitoring of magnetic nanoparticles," IEEE Transactions on Magnetics, vol. 51, pp. 1-5, 2015.

[18] T. Knopp and T. M. Buzug, "Magnetic particle imaging: an introduction to imaging principles and scanner instrumentation," Berlin, Heidelberg: Springer-Verlag, 2012.

[19] P. W. Goodwill and S. M. Conolly, "Multidimensional x-space magnetic particle imaging," IEEE Transactions on Medical Imaging, vol. 30, pp. 1581-1590, 2011.

[20] J. Weizenecker, J. Borgert and B. Gleich, "A simulation study on the resolution and sensitivity of magnetic particle imaging," Physics in Medicine and Biology, vol. 52, pp. 6363-6374, 2007.

[21] V. Schulz, M. Straub, M. Mahlke, S. Hubertus, T. Lammers, and F. Kiessling, "A field cancellation signal extraction method for magnetic particle imaging," IEEE Transactions on Magnetics, vol. 51, 2015.

[22] D. Eberbeck, F. Wiekhorst, S. Wagner, and L. Trahms, "How the size distribution of magnetic nanoparticles determines their magnetic particle imaging performance," Applied Physics Letters, vol. 98, p. $182502,2011$.

[23] K. P. Ivanov, M. K. Kalinina and L. Yui, "Blood flow velocity in capillaries of brain and muscles and its physiological significance," Microvascular Research, vol. 22, no. 2, pp. 143-155, 1981.

[24] M. Utkur, Y. Muslu and E. U. Saritas, "Relaxation-based viscosity mapping for magnetic particle imaging," Physics in Medicine and Biology, vol. 62, no. 9, pp. 3422-3439, 2017.

The English in this document has been checked by at least two professional editors, both native speakers of English. For a certificate, please see:

http://www.textcheck.com/certificate/y5VOH0 